\title{
A GESTÃO DA QUALIDADE EM SERVIÇOS DE INFORMAÇĀO COM BASE NA ISO 9000
}

\section{Valéria Martin Valls}

\section{Resumo}

Aborda a implantação da Gestão da Qualidade em Serviços de Informação com base nas normas da série ISO 9000, a partir de ampla revisão de literatura nacional e internacional, desde o início de sua incidência na literatura especializada até os dias atuais.

São apresentados e discutidos os principais benefícios, críticas e dificuldades da implantação deste tipo de modelo, traçando um panorama do tema, com o objetivo de apoiar os gestores de Serviços de Informação na sua compreensão e entendimento, contribuindo para a consolidação da Gestão da Qualidade na área de administração de Serviços de Informação.

\section{Palavras-chave}

Gestão da qualidade; Serviços de informação; ISO 9000.

\section{QUALITY MANAGEMENT OF INFORMATION SERVICES ACCORDING TO ISO 9000}

\begin{abstract}
The approach of ISO 9000 standards are used aiming at the implementation of Quality Management of Information Services from an extensive investigation on both, national and international literature, from earliest citations to those found on recent days. Presenting and discussing the benefits, concerns and difficulties of this kind of model, the role of the study is to support information services managers in their understanding and knowledge, which means the consolidation of Quality Management on Information System Management field..
\end{abstract}

\section{Keywords}

Quality management; Information services; ISO 9000. 


\section{INTRODUÇÃO}

O objetivo desse artigo é apresentar uma revisão de literatura nacional e internacional sobre a aplicação da Gestão da Qualidade em Serviços de Informação com base na ISO 9000, visando a apoiar seu entendimento e aplicação nos Serviços de Informação brasileiros, contribuindo para a ampliação das discussões sobre o tema, incluindo a apresentação de um panorama com os principais benefícios, críticas e dificuldades da aplicação deste modelo de gestão.

Em relação à literatura internacional especializada, as primeiras ocorrências sobre a aplicação de normas de garantia da qualidade em Serviços de Informação abordam a BS 5750, norma inglesa precursora da série de normas ISO 9000. Os primeiros trabalhos publicados por Dawson (1992), Ashcroft e Barton (1993), Jackson e Ashton (1993) e Wedlake (1993) analisam a introdução dos conceitos industriais para a prestação de serviços, destacando a dificuldade de interpretação dos requisitos dessa norma para a realidade dos serviços de informação. Há que se destacar que a BS 5750 estava muito direcionada para a garantia de qualidade de produtos, podendo considerar-se natural, portanto, esta fase inicial de dificuldade. Os trabalhos citados, em contrapartida, foram precursores e abriram espaço para colocar o tema em discussão, como podemos observar nos inúmeros trabalhos publicados na década seguinte. No Brasil, os primeiros trabalhos específicos sobre o tema foram publicados a partir de 2000, contendo estudos teóricos e relatos de experiência.

\section{OS TRABALHOS PRECURSORES SOBRE A APLICAÇÃO DA ISO 9000 EM SERVIÇOS DE INFORMAÇÃO}

Em 1994 Peter Brophy, citado no livro de Ashcroft e Barton (1993) como um pioneiro no estudo do tema, publica o artigo onde descreve a experiência da Biblioteca da University of Central Lancashire na implantação de um Sistema de Gestão da Qualidade com base nas normas ISO 9000, tendo sido a primeira biblioteca universitária na Inglaterra a obter esta conquista. Segundo o autor, no processo de implantação da Gestão da Qualidade, os responsáveis pela Biblioteca pesquisaram o conceito de "qualidade", especialmente sua aplicação em um serviço público e puderam identificar os possíveis avanços operacionais e estratégicos que o gerenciamento pela qualidade oportuniza, com ênfase na melhoria 
contínua. Neste contexto, as normas ISO 9000 ofereceram diretrizes consagradas internacionalmente para a Gestão da Qualidade, especialmente para o nível operacional. Através da implantação das normas ISO 9000 na Biblioteca, destaca-se a evolução que o Serviço de Informação obteve, especialmente em relação ao impacto na qualidade dos serviços prestados aos usuários. Um ponto a ser destacado neste artigo é a cultura da Universidade na qual a Biblioteca está inserida, pois o autor reforça que a implantação do Sistema de Gestão da Qualidade só foi possível pois a Universidade tem como missão transformar alunos em pesquisadores e os serviços de informação oferecidos têm um papel de destaque neste processo. Além disso, o próprio conceito de qualidade da Biblioteca, "atender às necessidades dos usuários com os recursos disponíveis", é bastante oportuno, pois um processo implantado em um Serviço de Informação só é viável com o apoio efetivo da Organização mantenedora. Adicionalmente, é dada ênfase ao atendimento das necessidades dos usuários, mesmo com a versão 1994 da ISO 9001, que ainda não reforçava este ponto. Neste sentido, no caso de uma Biblioteca Universitária, as necessidades dos usuários (especialmente dos alunos) está ligada à política educacional da Instituição Mantenedora, o que direciona o planejamento e a implantação dos serviços.

O relato de Peter Brophy (1994) é bastante enriquecedor, pois descreve (embora de forma sucinta) como o Sistema de Gestão da Qualidade foi implantado, suas dificuldades e as melhorias obtidas. O ponto alto do artigo é a desmistificação que o autor faz da padronização "dita" burocrática, colocando as diretrizes da ISO 9000 conceitualmente em seu devido lugar, ou seja, como uma diretriz a ser seguida, considerando sempre e essencialmente a cultura da Organização e suas características.

Também em 1994 Carl Johannsen publica o seu primeiro artigo sobre o tema, questionando se os conceitos da Gestão da Qualidade, especialmente relacionados às normas ISO, são ou não aplicáveis ao contexto das Bibliotecas. Para introduzir a discussão, o autor apresenta um panorama sobre as normas da série ISO 9000, incluindo uma breve revisão de literatura. A partir destes conceitos, a aplicabilidade da teoria é analisada no âmbito das Bibliotecas. A conclusão do autor é de que as normas ISO 9000 são aplicáveis aos Serviços de Informação, porém ressalta que os objetivos e a maneira como a implementação é conduzida são elementos importantes para que os reais objetivos da Gestão da Qualidade sejam atingidos. Como reforça Johannsen, por exemplo, se a implementação for realizada mecanicamente, sem 
o entendimento da filosofia da norma, certamente o Sistema de Gestão da Qualidade será burocrático e pouco útil aos gestores envolvidos. Além disso, são apresentadas quatro recomendações baseadas no estudo de caso do "Projeto Nórdico", que envolveu a aplicação dos conceitos de Qualidade Assegurada em Bibliotecas:

$1^{\mathrm{o}}$ A análise dos requisitos a partir da realidade dos processos implantados. As alterações nas atividades devem ser realizadas respeitando sua natureza e não somente visando atender aos requisitos da norma;

$2^{o}$ É crucial que a seleção e utilização dos instrumentos utilizados no Sistema da Qualidade sejam baseados na análise e entendimento das necessidades dos clientes. É preciso evitar que a implementação traga burocracia e comprometa os padrões de qualidade já conquistados;

$3^{\text {o }}$ É importante ter em mente que o Sistema da Qualidade implantado deve estar alinhado à cultura da Organização e que a interface com os colaboradores deve ser feita de maneira cuidadosa, para que eles colaborem com as mudanças pretendidas;

$4^{\circ}$ A norma ISO 9001 não deve ser vista somente como um guia para a conquista de padrões de qualidade. A implantação da Gestão da Qualidade é um processo complexo, que envolve muitas etapas, principalmente as referentes a cultura da qualidade e o entendimento de seus princípios e filosofia, para que os objetivos sejam efetivamente cumpridos.

O panorama traçado por Johannsen é bastante contemporâneo, principalmente no item referente às críticas. Embora a versão da norma tenha sido revisada, muitas Organizações ainda implantam um Sistema de Gestão da Qualidade sem um entendimento claro do seu real objetivo. O artigo possibilita uma reflexão bastante oportuna sobre o assunto e pode ser complementado por outros artigos publicados pelo autor, em 1995 e 1996, também relatando a experiência prática originada do Projeto Nórdico (1993/94).

Complementando esta primeira fase de estudo sobre o tema, Elisa Garcia-Morales divulga em 1994, em evento realizado na Espanha, uma comunicação onde é traçado um panorama sobre a aplicação da filosofia da Gestão da Qualidade em Serviços de Informação, com ênfase na revisão de mentalidade em relação às necessidades dos usuários, destacando a dificuldade em obter informações precisas sobre as reais expectativas dos clientes, que servem de parâmetro 
para a estruturação dos processos de trabalho, indispensáveis para a obtenção de níveis elevados de qualidade. É apresentada uma análise histórica sobre a relação entre os Serviços de Informação e os seus usuários, principalmente reforçando a necessidade de mudança de mentalidade dos provedores de serviços de informação, que não devem somente nomear mas efetivamente enxergar os usuários como clientes. Esta análise é feita de forma bastante realista, destacando que nem todos os usuários serão atendidos com padrões máximos de qualidade. É introduzido o conceito de clientes-chave e de níveis máximos de qualidade que o Serviço de Informação pode alcançar, abordagem muita oportuna, principalmente considerando os recursos disponíveis (que nem sempre são os necessários para atender a todos os objetivos planejados).

Outro ponto estudado refere-se à necessidade de estabelecimento e implantação de indicadores ou outras formas de medição, capazes de demonstrar em que grau os serviços cumprem os objetivos planejados e se estão, de fato, atendendo às expectativas dos clientes. $\mathrm{O}$ estabelecimento de indicadores de desempenho é uma atividade conhecida dos bibliotecários, principalmente sob o enfoque de estatísticas e medições, usuais em Serviços de Informação. Neste sentido, estabelece-se uma diferenciação entre os indicadores de atividades e os indicadores de qualidade, sendo que o segundo tipo torna-se uma importante ferramenta de gestão, sendo a base para a racionalização e redistribuição dos recursos disponíveis. Além disso, a autora também apresenta o que ela nomeia de "Quatro regras fundamentais da Qualidade aplicadas aos Serviços de Informação", que são:

$1^{\circ}$ A qualidade é o primeiro passo, uma vez que somente essa forma de gestão produz benefícios a longo prazo;

$2^{\circ}$ O mercado deve "entrar" nos Serviços de Informação, principalmente sob o aspecto do atendimento aos clientes;

$3^{o} \mathrm{O}$ ambiente interno do Serviço de Informação deve orientar-se interna e externamente pelas necessidades dos clientes;

$4^{\circ}$ Os aspectos relacionados à qualidade devem ser mensuráveis.

O trabalho apresentado por Elisa Garcia-Morales (1994) é muito elucidativo, pois apresenta pontos básicos referentes aos fundamentos da Gestão da Qualidade aplicados diretamente ao ambiente dos Serviços de Informação, de uma forma didática e buscando sempre o 
contraponto entre estes fundamentos e a realidade deste tipo particular de prestação de serviços, tendo como pano de fundo uma visão realista e pragmática.

Essa visão pragmática também pode ser observada no livro de Ellis e Norton (1996), que aborda, através da experiência de seus autores na implantação do Sistema de Gestão da Qualidade do Management Information Centre, na Inglaterra, noções básicas sobre a Gestão da Qualidade, os requisitos da norma ISO e sua aplicação (com exemplos aplicados à realidade da Biblioteca), a documentação dos processos de trabalho, as auditorias da qualidade, a implantação de indicadores de desempenho, o gerenciamento das mudanças acarretadas com a implantação da Gestão da Qualidade, o processo de certificação e os Organismos certificadores da Inglaterra e, concluindo, o gerenciamento do Sistema após a certificação. O objetivo básico do livro é ser um guia prático para auxiliar na implantação de Sistemas de Gestão da Qualidade em Bibliotecas e através dos exemplos apresentados e da experiência de seus autores, representa uma importante contribuição para a literatura da área.

\section{O RELATO DE EXPERIÊNCIAS PRÁTICAS}

Nota-se, como foi abordado no item anterior, uma tendência marcante na literatura analisada, a de abordar o tema através da apresentação de casos práticos de implantação das diretrizes das normas ISO 9000 em Serviços de Informação. O Quadro a seguir ilustra esta tendência, com experiências relatadas entre 1996 e 2004, apresentadas em ordem cronológica: 


\section{QUADRO 1 - SISTEMAS DE GESTÃO DA QUALIDADE IMPLANTADOS EM SERVIÇOS DE INFORMAÇÃO}

\begin{tabular}{|c|c|c|c|}
\hline $\begin{array}{l}\text { AUTOR(ES) /ANO DE } \\
\text { PUBLICAÇÃO }\end{array}$ & DESCRIÇÃO & PAÍS & REFERÊNCIA \\
\hline VASSEUR (1997) & $\begin{array}{l}\text { Centro de Documentação da } \\
\text { SOGREAH }\end{array}$ & França & ISO $9001: 1994$ \\
\hline COLES (1999) & $\begin{array}{l}\text { Sistema de Bibliotecas Públicas de } \\
\text { Wirral }\end{array}$ & Inglaterra & ISO $9002: 1994$ \\
\hline DATE; GOKHALE (1999) & $\begin{array}{l}\text { Grupo de treze bibliotecas industriais } \\
\text { de Mundai }\end{array}$ & Índia & ISO 9004-2 : 1992 \\
\hline BALAGUÉ MOLA $(2000,2001,2002 b)$ & $\begin{array}{l}\text { Sistema de Bibliotecas da UAB - } \\
\text { Universidade Autônoma de Barcelona }\end{array}$ & Espanha & ISO $9002: 1994$ \\
\hline DUARTE BARRIONUEVO (2000) & $\begin{array}{l}\text { Sistema de Bibliotecas da UCA - } \\
\text { Universidade de Cadiz }\end{array}$ & Colômbia & $\begin{array}{l}\text { Balanced Scorecard } \\
\text { ISO } 9001: 2000\end{array}$ \\
\hline $\begin{array}{l}\text { GRABOLOSA SELLABONA; VICENS } \\
\text { PERPINYÀ(2000) }\end{array}$ & $\begin{array}{l}\text { Centro de Documentação do Parque } \\
\text { Natural da Zona Vulcânica da Garrotxa }\end{array}$ & Espanha & ISO $9002: 1994$ \\
\hline KRESTEL (2000) & $\begin{array}{l}\text { Centro de Informação sobre Patentes de } \\
\text { Nuremberg }\end{array}$ & Alemanha & ISO $9001: 1994$ \\
\hline TÉRMENS GRAELLS (2000) & $\begin{array}{l}\text { Sistema de Bibliotecas da UAB - } \\
\text { Universidade Autônoma de Barcelona }\end{array}$ & Espanha & ISO $9002: 1994$ \\
\hline SARDELLI (2001) & Biblioteca Nacional Central de Firenze & Itália & ISO $9002: 1994$ \\
\hline BARBÊDO; TURRIONI, (2003) & $\begin{array}{l}\text { Biblioteca Mário Henrique Simonsen } \\
\text { da Fundação Getúlio Vargas (FGV-RJ) }\end{array}$ & Brasil & ISO $9001: 2000$ \\
\hline GIRALDO ARREDONDO (2003) & $\begin{array}{l}\text { Departamento de Cultura e Bibliotecas } \\
\text { de Comfenalco Antioquia }\end{array}$ & Colômbia & ISO $9001: 2000$ \\
\hline LÓPEZ CALLE (2003) & $\begin{array}{l}\text { Departamento de Bibliotecas da } \\
\text { Universidade de Antioquia }\end{array}$ & Colômbia & ISO $9001: 2000$ \\
\hline
\end{tabular}

Dos trabalhos citados, ressalta-se o artigo divulgado por Claudia Giraldo Arredondo (2003), que relata a experiência do Departamento de Cultura e Bibliotecas de Comfenalco em Antioquia, na Colômbia. Com o objetivo de compartilhar sua experiência, a autora apresenta um panorama geral sobre Sistemas da Qualidade, um breve histórico sobre o Departamento de Cultura e Bibliotecas e o projeto de Gestão da Qualidade, até a obtenção do certificado, incluindo a contribuição das Bibliotecas nesta implantação. As Bibliotecas deste Departamento foram organizadas tendo como base as diretrizes e princípios do Manifesto da Unesco para Bibliotecas Públicas, embora a Comfenaldo seja uma ONG (Organização Não Governamental), de apoio social mantida por entidades privadas. A certificação da Comfenaldo envolveu todos os departamentos do grupo (Hotel, Bibliotecas, Instituto de Educação, Agência de Turismo, etc), e a autora destaca que as Bibliotecas, neste contexto, se somaram ao esforço de certificação. Segundo sua opinião, uma biblioteca pública independente não poderia assumir isoladamente um projeto como este, principalmente por não ter a exigência formal quanto à certificação e também por não dispor de meios e recursos para implantar um Sistema de Gestão da Qualidade em sua plenitute (GIRALDO ARREDONDO, 2003). Além disso, são apresentados os requisitos da ISO 9001 versão 2000 e sua aplicação no Departamento de Cultura e Bibliotecas, ilustrando de maneira bastante 
didática como foi feita a interpretação dos requisitos da norma para sua aplicação em um Serviço de Informação.

Outra experiência colombiana é relatada em López Calle (2003), que apresenta a experiência do Departamento de Bibliotecas da Universidade de Antioquia na implantação e certificação de seu Sistema de Gestão da Qualidade baseado na ISO 9001 : 2000. Esta comunicação, além de apresentar os aspectos conceituais da Gestão da Qualidade e a metodologia utilizada na Universidade, apresenta dados estatísticos de melhoria em relação à satisfação do usuário, satisfação da equipe e resultados gerais da Biblioteca, como por exemplo: índices de reclamação, níveis de solução de reclamações, índices de funcionários treinados, incremento de processamento técnico, de empréstimo de materiais e de cursos ministrados aos usuários, como forma de exemplificar as melhorias obtidas a partir da implantação do Sistema.

Adicionalmente, outra autora que merece destaque é Núria Balagué Mola (2000, 2001, 2002a e 2002b) que descreve a experiência de implantação das normas ISO 9000 no Serviço de Bibliotecas da Universidade Autonôma de Barcelona. Na comunicação "A aplicação das normas ISO nos centros de documentação: uma oportunidade de melhoria" (BALAGUÉ MOLA, 2002a) são descritos detalhadamente os principais passos para a implantação de um Sistema de Gestão da Qualidade baseado nas normas ISO 9001 : 2000 em Serviços de Informação, que são: apresentação do projeto, diagnóstico preliminar, planejamento e organização das atividades, preparação da documentação do Sistema de Gestão da Qualidade, implantação dos processos documentados, pré-auditoria de certificação, certificação do Sistema de Gestão da Qualidade e manutenção e melhoria do Sistema após a certificação. Com riqueza de detalhes a autora apresenta as dificuldades e questionamentos que foram sendo superados ao longo do processo, além de apresentar algumas vantagens e desvantagens que podem advir deste tipo de projeto, o que enriquece e muito a análise apresentada.

Além dos trabalhos com esta abordagem eminentemente prática, a literatura especializada conta também com estudos teóricos, onde destacam-se em ordem cronológica, Jha et al. (1997), Pacios Lozano (1997), Abreu e Andalia (1998), Mulye e Deshpande (1998), Portuondo Sánchez (1998), Dobson e Ernst (1999), Pelizzari (1999), Sardelli (1999), Ramesh (2000), Glowacka (2002), Gómez Hernandez (2002), Selvi (2002), Barbêdo (2004), Martins e Lotti (2004) e Valls (2005). 


\section{PANORAMA SOBRE A APLICAÇÃO DA GESTÃO DA QUALIDADE COM BASE NA ISO 9000 EM SERVIÇOS DE INFORMAÇÃO}

A partir da análise dos trabalhos citados, podemos traçar uma visão geral sobre a aplicabilidade dos conceitos e fundamentos das normas ISO 9000 em Serviços de Informação, exemplificadas nos itens a seguir. Para elaborar este panorama, foram considerados prioritariamente os trabalhos que analisam a versão 2000 da ISO 9001, por entender-se que muitas críticas feitas à versão anterior da norma foram sanadas a partir da nova versão. Além disso, foram considerados os oito princípios da Gestão da Qualidade, apresentados na NBR ISO 9000 (ASSOCIAÇÃO BRASILEIRA DE NORMAS TÉCNICAS, 2000, p. 2), para contextualizar os benefícios identificados:

a) Principais benefícios identificados na literatura analisada (ordenados pelos oito princípios da Gestão da Qualidade):

\section{Foco no cliente}

- Conversão das necessidades subjetivas dos usuários em processos documentados;

- Estabelecimento de processos para recepção e tratamento das manifestações dos usuários (elogios, críticas e sugestões);

- Implantação de comitês de usuários e outras formas de aproximação com os fornecedores de serviços de informação;

- Introdução do conceito de cliente interno, gerando mais sinergia e integração entre as equipes;

- Maior aproximação e interação com os usuários, identificando suas reais necessidades de informação e expectativas em relação aos serviços prestados.

\section{Liderança}

- Ampliação do papel da Direção e demais lideranças setoriais, responsáveis pela motivação da equipe e pelo planejamento, implantação e melhoria do Sistema de Gestão da Qualidade; 
- Ênfase no capital humano das bibliotecas, como recurso de sustentação dos processos implantados, que dependem diretamente da postura e motivação dos líderes;

- Fortalecimento das lideranças.

\section{Envolvimento de pessoas}

- Compartilhamento de conhecimentos e experiências individuais;

- Elevação da moral da equipe, em razão do seu envolvimento com o Sistema de Gestão da Qualidade e com o feedback dos usuários;

- Identificação de talentos e líderes natos;

- Maior participação dos membros da equipe nas decisões relativas aos serviços (Enfoque democrático);

- Mudança da cultura organizacional, viabilizando novas oportunidades profissionais e pessoais para os membros da equipe;

- Redução da tensão entre os funcionários, pois eles sabem claramente o que a Organização espera deles;

- Treinamento de toda a equipe envolvida direta ou indiretamente com o atendimento.

\section{Abordagem de processo}

- Oportunidade de simplificação das rotinas técnicas e administrativas, com o planejamento e implantação de processos integrados;

- Padronização das atividades, através da documentação elaborada e treinamento dos envolvidos;

- Prioridade nos processos que originam os produtos e serviços.

\section{Abordagem sistêmica para a gestão}

- Atividades setoriais passam a ser de conhecimento de toda a equipe, de maneira transparente e compreensível, facilitando o entendimento das inter-relações e coresponsabilidades; 
- Definição clara das funções e responsabilidades de cada membro da equipe, onde todos passam a ser diretamente responsável pelo Sistema de Gestão da Qualidade;

- Integração com as demais áreas da Instituição mantenedora;

- Maior integração das equipes técnicas, de atendimento, administrativas, etc;

- Monitoramento da qualidade de todos os produtos e serviços implantados, de forma sistêmica e objetiva.

\section{Melhoria contínua}

- Elevação da credibilidade do serviço e dos profissionais de informação perante a instituição mantenedora e os usuários;

- Estabelecimento de critérios para o desenvolvimento de coleções (acervo físico e digital);

- Estabelecimento da cultura da melhoria contínua, incluindo a utilização das ferramentas da qualidade para detectar, prevenir e corrigir falhas;

- Estabelecimento da Política do Serviço de Informação;

- Implantação das auditorias internas e externas como forma de analisar constantemente o desempenho dos processos;

- Maior agilidade na realização das atividades, em razão dos ciclos mais otimizados e integrados;

- Maior controle dos custos e despesas;

- Maior planejamento e priorização das tarefas e atividades;

- Melhor organização do acervo;

- Melhoria da qualidade dos dados e informações fornecidos, em razão do aperfeiçoamento do processo de comunicação;

- Melhoria na divulgação e promoção dos produtos e serviços;

- Obtenção de recursos, com a comprovação das necessidades baseada em fatos concretos;

- Otimização do uso dos recursos disponíveis;

- Potencialização da cooperação interbibliotecária;

- Redução de custos, principalmente relacionados ao retrabalho e ao desperdício;

- Suspensão de atividades e rotinas burocráticas que não agreguem valor ao sistema implantado; 
- Unificação de critérios.

\section{Abordagem factual para tomada de decisão}

- Implantação de indicadores da qualidade e gestão baseada em fatos e dados;

- Implantação de mecanismos de prevenção e correção;

- Implantação de mecanismos de supervisão e controle;

\section{Benefícios mútuos nas relações com os fornecedores}

- Maior relacionamento com os fornecedores de produtos e serviços.

b) Principais dificuldades encontradas e críticas a este tipo de modelo, identificados na literatura analisada:

- Ausência de apoio da Alta Direção da instituição mantenedora;

- Ausência de recursos humanos e tecnológicos para a implantação de todos os processos necessários;

- Caráter das normas ISO ainda é eminentemente industrial e sua aplicação em prestação de serviços de informação é muito difícil e complicada;

- Cerceamento da criatividade e da inovação quando do estabelecimento da padronização de atividades;

- Complexidade do processo de implantação de um Sistema de Gestão da Qualidade subestimado, envolvendo iniciativas empíricas e sem fundamentação teórica, causando descontinuidade e frustração dos envolvidos;

- Diferentes perfis e conceitos pessoais dos funcionários, inviabilizando muitas vezes a padronização;

- Dificuldade em documentar as atividades, em razão da "tradição oral" dos Serviços de Informação;

- Dificuldade em implantar mecanismos de comunicação com os usuários;

- Dificuldade em incorporar o Sistema de Gestão da Qualidade na vida diária do Serviço de Informação e na própria administração;

- Dificuldade em interpretar os requisitos da norma para a realidade da prestação de serviços de informação; 
- Equipe satisfeita com o status quo do Serviço de Informação, não sendo receptiva a mudanças;

- Excesso de documentação gerada em algumas atividades;

- Excesso de medições e controles;

- Imposição por parte do dirigente do Serviço de Informação, sem esclarecimento e motivação da equipe;

- Imposição de sistemas prontos e pré-elaborados, sem respeitar a cultura do Serviço de Informação (especialmente quando é contratada consultoria externa);

- Implantação da Gestão da Qualidade isoladamente no Serviço de Informação, sem uma Política da Qualidade definida e implantada na Instituição mantenedora;

- Inibição da flexibilidade, muitas vezes burocratizando o atendimento;

- Postura passiva dos dirigentes de Serviços de Informação em relação às críticas e sugestões dos usuários, inviabilizando uma gestão focada no cliente;

- Utilização da ISO 9000 como um fim, não como um meio para alcançar a qualidade do serviço;

- Variedade de serviços implantados.

Tendo como base os artigos, estudos acadêmicos, comunicações e livros analisados neste estudo, podemos concluir que a implantação da Gestão da Qualidade em Serviços de Informação com base na série ISO 9000 é uma estratégia viável e pode ser utilizada pelos dirigentes destes serviços em iniciativas ligadas à melhoria da qualidade dos serviços e produtos oferecidos e elevação dos níveis de satisfação dos usuários.

\section{CONSIDERAÇÕES FINAIS}

Como foi apresentado, a literatura descreve várias experiências práticas e estudos teóricos que analisam esta aplicação e demonstram, mesmo de forma genérica, como um projeto deste tipo pode ser conduzido. Obviamente, cada Organização possui características típicas e o ambiente interno e externo de cada uma delas pode diferir, auxiliando ou até mesmo dificultando a implantação de projetos ligados à Gestão da Qualidade; todavia, foi observada uma predisposição para utilizar os princípios definidos na série de normas ISO 9000 como parâmetro para guiar e apoiar iniciativas de melhoria da qualidade, o que, por si só, já é um benefício importante, já que as normas são consagradas internacionalmente como um guia 
para a implantação da Gestão da Qualidade em organizações de diferentes tipos e portes, como foi visto anteriormente.

Há que se destacar, nesse contexto, que o crescimento do número de certificados ISO 9000 no Brasil cresceu exponencialmente, saindo de 18 certificados emitidos em 1990 para 5.518 em 2004, segundo dados oficiais da ABNT (PERSPECTIVAS, 2004, p. 7). Esse panorama certamente favoreceu a opção de muitos Serviços de Informação pelas normas da família ISO 9000, que além de ser um padrão internacionalmente consagrado, apresentam os requisitos mínimos para a implantação de um Sistema de Gestão da Qualidade, com o objetivo de assegurar que produtos, serviços e processos cumpram os requisitos especificados. Embora a norma não defina como o processo deve ser implantado, define claramente os objetivos de cada processo e sua relação com o sistema como um todo. Desta forma,

\begin{abstract}
pode-se afirmar que bibliotecas necessitam buscar novas formas de incluir o cliente / usuário na filosofia dos serviços que utilizam e a aplicação de um sistema de gestão da qualidade neste setor pode contribuir para o desenvolvimento de atividades atendendo as necessidades de seus usuários, além de uma melhor organização interna, definição clara e documentada das atividades e responsabilidades e de sua equipe e o fortalecimento da competência e qualidade do trabalho. [...] Sendo que a nova versão da ISO 9000 (2000) preocupa-se com o foco no cliente e o gerenciamento de Bibliotecas busca uma melhoria no atendimento ao usuário, espera-se que a implementação da ISO 9000 : 2000 em bibliotecas signifique a garantia da qualidade em todos os processos para satisfação deste usuário (BARBÊDO; TURRIONI, 2003).
\end{abstract}

Especificamente sobre a opção dos Serviços de Informação pelas diretrizes da série ISO 9000 em detrimento de outros modelos disponíveis, entende-se que este fato em si não é muito relevante, pois o mais importante é considerar a norma como um meio para atingir os objetivos planejados, não um fim em si mesmo. Muitas Organizações optam por uma certificação com base na NBR ISO 9001, por exemplo, somente para fins de divulgação e marketing e neste caso, certamente o Sistema de Gestão da Qualidade implantado tende a se transformar em um problema para a Organização, especialmente sua manutenção pós certificação. Em contrapartida, muitas outras Organizações optam pelo modelo proposto nas normas da família ISO como um auxílio para seus projetos de melhoria.

É importante ressaltar também que optar pelas diretrizes da ISO 9000 não quer dizer, obrigatoriamente, buscar a certificação do Sistema de Gestão da Qualidade por um Organismo Certificador, que demanda recursos e principalmente o envolvimento de toda a Organização. Neste sentido, em muitos Serviços de Informação o objetivo principal de iniciar um Programa de Qualidade não é a certificação em si ou seu valor de marketing, mas, principalmente, o 
anseio de melhorar seus processos gerenciais e os serviços ao cliente (JOHANNSEN, 1996). Essa quebra de paradigma é muito importante para desmistificar um pouco o temor que alguns profissionais têm em utilizar a norma, com receio de que ao iniciar este projeto estejam em um caminho sem volta, ou seja, envolvendo-se em um contrato com o Organismo Certificador e até mesmo criando uma expectativa nos usuários e demais envolvidos. Antes de tudo, planejar e implantar um Sistema de Gestão da Qualidade com base nas normas ISO 9000 é uma escolha extremamente admissível para os Serviços de Informação brasileiros, principalmente considerando-se as várias experiências de sucesso relatadas na literatura internacional, dentre as quais destaca-se a de Nora Helena López Calle(2003):

\begin{abstract}
Os diversos modelos administrativos oferecem instrumentos e ações para conduzir a mudança nas Organizações, um deles é a Gestão da Qualidade baseada na ISO 9000. A importância desta série de normas, independente da opção pela certificação, é a possibilidade de reorganizar a biblioteca em aspectos como planejamento de uma estratégia de melhoria contínua; motivar, integrar e responsabilizar todos os funcionários; contar com uma melhor organização, planejamento e coordenação interna; aumentar a fidelidade dos usuários e economizar despesas através de métodos adequados.
\end{abstract}

Outro ponto importante é que dispor de um Sistema de Gestão da Qualidade implantado não quer dizer que o Serviço de Informação é infalível, que a equipe é perfeita e que os usuários serão eternamente satisfeitos. Longe disso, o grande objetivo é manter os processos sob controle, conhecendo suas características e limitações e dispondo de informações para a correção de eventuais desvios, dentre outros objetivos já citados ao longo deste trabalho. Pode-se afirmar, resumidamente, que o grande objetivo desta implantação é a previsibilidade, ou seja, a possibilidade de prever tanto as necessidades e recursos, quanto os eventuais problemas ou desvios ao longo dos processos. Nesta sutil contradição, de propor um sistema aparentemente complexo baseado em conceitos básicos é que reside um dos maiores atrativos da NBR ISO 9001. Particularmente nos Serviços de informação,

Um Sistema da Qualidade com base na norma ISO tem que ser capaz de demonstrar a todo momento que seus requisitos são cumpridos. Não se trata de fazer mais coisas em menos tempo, nem fazer de um modo espetacular; trata-se de descrever o que se faz, fazer e demonstrar a qualquer momento que se fez tal como está descrito na documentação (BALAGUÉ MOLA, 2001, p. 14).

Desta forma, entender claramente o que cada requisito propõe e descrever o Sistema de Gestão da Qualidade adequado para cada Serviço de Informação é o primeiro passo a ser alcançado. Muitas iniciativas são implantadas sem sucesso porque o desenho do Sistema é copiado de uma outra realidade, como se uma fórmula de sucesso pudesse ser repetida. É claro que analisar outras experiências é uma técnica eficaz, pois evita que problemas sejam 
repetidos e principalmente permite que boas idéias sejam renovadas, mas o planejamento de um Sistema de Gestão da Qualidade depende diretamente das características de cada Organização. Neste sentido, "talvez a única receita válida para a implementação da qualidade seja aquela que afirma não existirem receitas válidas” (VERGUEIRO, 2002, p. 118).

Além disso, uma das grandes críticas às normas ISO 9000 é que elas são burocráticas e que engessam a equipe, impedindo até mesmo a sua flexibilidade e criatividade, já que a padronização dos processos uniformiza as atividades, com o estabelecimento dos documentos e demais padrões. Especialmente nos Serviços de Informação, esta visão é também contraditória, pois a Biblioteconomia é uma ciência eminentemente prescritiva, que possui regras e códigos seculares para a análise e organização de dados, informações e conhecimentos. Entende-se que a ampliação da tradição de padronização dos Serviços de Informação, dos setores técnicos para os setores de atendimento é possível e necessária, pois a satisfação do cliente está alicerçada em elementos subjetivos e quanto mais organizado e previsível um processo, maior tende a ser a percepção de qualidade dos clientes deste serviço.

Neste sentido, considera-se este um importante passo na evolução das teorias administrativas aplicadas aos Serviços de Informação, oportunizando o exercício de práticas gerenciais contemporâneas, fundamentadas em posturas profissionais e visão de mercado, entendido aqui em seu sentido mais básico: o atendimento aos usuários, razão de ser de qualquer Serviço de Informação.

\section{REFERÊNCIAS}

ABREU, M. C.; ANDALIA, R. C. Gerencia total de la calidad en las organizaciones. ACIMED, Habana, v. 6, n. 2, p. 79-92. 1998. Disponível em: $<$ http://eprints.rclis.org/archive/00002044/01/aci02298.pdf>. Acesso em: 31 dez. 2004.

ASHCROFT, M.; BARTON, D. Quality management: towards BS 5750. Stamford: Capital Planning Information, 1993. $34 \mathrm{p}$.

ASSOCIAÇÃO BRASILEIRA DE NORMAS TÉCNICAS. NBR ISO 9000: Sistemas de gestão da qualidade - fundamentos e vocabulário. Rio de Janeiro, 2000. 26 p.

BALAGUÉ MOLA, N. Aplicación de las normas ISO 9000 en bibliotecas: la experiencia del Servicio de Bibliotecas de la Universidad Autónoma de Barcelona. CalidadLatina.Com, Barcelona, n. 14, oct. 2001. Disponível em: <http://www.calidadlatina.com/pub/014-OCT01.pdf>. Acesso em: 30 dez. 2004. 
BALAGUÉ MOLA, N. La aplicación de las normas ISO 9000 en los centros de documentación: una oportunidad de mejora. In: SEMINARIO SOBRE CENTROS DE DOCUMENTACIÓN EN MATERIA DE MEDIO AMBIENTE Y ESPACIOS NATURALES PROTEGIDOS, 2002, Segovia, Ponencias... Centro Nacional de Educación Ambiental de Valsaín, 2002a. Disponível em: $<$ http://www.mma.es/educ/ceneam/pdf_w/balague1.pdf $>$. Acesso em: 30 dez. 2004. (v. não paginado).

Implementación de un sistema de calidad basado en la norma ISO 9002 en el servei de biblioteques de la Universitat Autònoma de Barcelona. Bellaterra, may 2000. 15 p. Disponível em: <http://prisma.psc.uva.es/jornadas/Consultas/Word/Docu22.doc $>$. Acesso em: 30 dez. 2004.

Implementing ISO 9000 standards in a University Library: The quality system at the Universitat Autònoma de Barcelona Library System. In: IFLA. Education and research for marketing and quality management in libraries. Munchen: Saur, 2002b. p. 145-156.

BARBÊDO, S. A. D. Sistema de gestão da qualidade em serviços: estudo de caso em uma biblioteca universitária. 2004. 134 f. Dissertação (Mestrado em Engenharia de Produção) Universidade Federal de Itajubá, Itajubá, 2004. Disponível em: $<$ http://www.ppg.efei.br/cpgp/Dissertacao/2004/89.pdf>. Acesso em: 26 nov. 2004.

.; TURRIONI, J. B. Sistema de gestão da qualidade e um modelo de integração estrutural em bibliotecas: análise comparativa em dois estudos de caso. In: SIMPÓSIO DE ENGENHARIA DE PRODUÇÃO, 10., 2003, Bauru. Anais... Bauru: UNESP, 2003. Disponível em:

$<$ http://www.simpep.feb.unesp.br/anais10/gestaoqualidprodutividade/arq01.PDF>. Acesso em: 24 nov. 2004. (v. não paginado).

BROPHY, P. The quality program of the library and learning resources service at the University of Central Lancashire. Inspel, Berlin, v. 28, n. 2, p. 240-7, 1994. Disponível em: $<$ http://www.fh-potsdam.de/ IFLA/INSPEL/94-2brpe.pdf>. Acesso em: 21 dez. 2004.

COLES, J. A wirral si sperimenta il sistema qualità. Biblioteche Oggi, Milano, v. 17, n. 6, p. 6-13, lug./ago. 1999. Disponível em:

<http://www.bibliotecheoggi.it/1999/19990600601.pdf>. Acesso em: 21 dez. 2004.

DATE, D. A.; GOKHALE, P. A. Implementation of ISO 9000 in industrial libraries: a case study. Library Science with a slant to Documentation and Information Studies, Bangalore, v. 36, n. 2, p. 65-72, june 1999.

DAWSON, A., Quality first: the Taywood Information Centre and BS 5750. Aslib Information, London, v. 20, n. 3, p. 112-113, 1992.

DOBSON, C.; ERNST, C. ISO 9000: the librarian's role. Information Outlook, Washington, p. 25-28, june 1999. 
DUARTE BARRIONUEVO, M. Searching excellence in the Library System of the University of Cadiz. In: INTERNATIONAL CONFERENCE ON QUALITY MANAGEMENT IN ACADEMIC LIBRARIES. Bydgoszcz, 2000. Proceedings... Disponível em: <http://ebib.oss.wroc.pl/matkonf/atr/miquel_pl.html>. Acesso em: $30 \mathrm{dez}$. 2004.

ELLIS, D.; NORTON, B. Implementing BS EN ISO 9000 in libraries. London: Aslib, 1996. $138 \mathrm{p}$.

GARCIA-MORALES, E. H. Gestión de calidad, análisis de necesidades de los usuarios e indicadores para servicios de información y documentación. In: JORNADAS DE INFORMACIÓN Y DOCUMENTACIÓN DE CIENCIAS DE LA SALUD, 5., 1994. Anais... Palma de Mallorca, 1994. Disponível em: <http://www.abadib.es/5jornadassalud/comunicacion37.htm>. Acesso em: $31 \mathrm{dez}$. 2004. (v. não paginado).

GIRALDO ARREDONDO, C. M. Modelo de aseguramento de la calidad em Bibliotecas: el caso del Departamento de Cultura y Bibliotecas de Comfenaldo Antioquia. Revista Interamericana de Bibliotecologia, Medellin, v. 26, n. 1, p. 125-152, enero/junio 2003.

GLOWACKA, E. An introduction into quality assurance and total quality management with reference to library and informations institutions. EBIB, Bydgoszcz, n.1, 2002. Disponível em: <http://ebib.oss.wroc.pl/english/grant/glowacka.php>. Acesso em: 4 jan. 2005.

GÓMEZ HERNÁNDEZ, J. A. Gestion de bibliotecas. Calidad y Evaluacion, Murcia: 2002. p. 65-76. Disponível em: <http://gtil.edu.um.es:8080/jgomez/bibgen/intranet/04gestionb.PDF>. Acesso em: 30 dez. 2004.

GRABOLOSA SELLABONA, M.; VICENS PERPINYÀ, J. Funcionament d'un centre de documentació amb la ISO 9002: el cas del Centre de Documentació del Parc Natural de la Zona Volcànica de la Grarrotxa. Biblioteca Informacions, Barcelona, v. 22, p. 14-18, 2000. Disponível em: <http://www.bib.uab.es/pub/bibinf/bi-22.pdf>. Acesso em: 30 dez. 2004.

JACKSON, P.; ASHTON, D. Implementing quality through BS 5750 (ISO 9000). London: Kogan Page: 1993. 225p.

JHA, K. N. et al. Application of ISO-9000 for library and information services. Annals of Library Science and Documentation, New Delhi, v. 44, n. 3, p. 81-85, 1997.

JOHANNSEN, C. G. Application of quality management in the nordic countries: results from the nordic quality management project. FID News Bulletin, Hague, v. 45, n. 5, p. 149-52, 1995.

Can the ISO-Standards on Quality Management be useful to libraries and how? Inspel, Berlin, v. 28, n. 2, p. 227-39, 1994. Disponível em: <http://www.fhpotsdam.de/ IFLA/INSPEL/94-2joca.pdf>. Acesso em: 21 dez. 2004.

. ISO 9000: a managerial approach. Library Management, Bradford, v. 17, n. 5, p. 14-24, 1996. 
KRESTEL, H. Certification of a patent information centre according to DIN EN ISO 9001. World Patent Information, Oxford, v. 22, p. 19-22, 2000.

LÓPEZ CALLE, N. H. El sistema de gestion de la calidad, una filosofia de trabajo en el Departamento de Bibliotecas de la Universidad de Antioquia. In: ENCUENTRO INTERNACIONAL DE BIBLIOTECAS - LA BIBLIOTECA PARA UNA UNIVERSIDAD DE EXCELENCIA: HUMANIZACIÓN, INNOVACIÓN Y COOPERACIÓN, 2003, Medellín, Universidad de Antioquia, 2003. (v. não paginado).

MARTINS, V. dos S. G.; LOTTI, Luciane Politi. Guia para implantação da norma NBR ISO 9001:2000 em Bibliotecas. In: SEMINÁRIO NACIONAL DE BIBLIOTECAS UNIVERSITÁRIAS, 13., 2004, Natal. Anais... Natal: BCZM, 2004.

MULYE, R.; DESHPANDE, N. J. A review of quality systems and its applications to libraries. Library Science with a slant to Documentation and Information Studies, Bangalore, v. 35, n. 1, p. 3-20, 1998.

PACIOS LOZANO, A. R. ISO 9000 and the total quality management models. Library Management, Bradford, v. 18, n. 3, p. 148-150, 1997.

PELIZZARI, E. Qualità in biblioteca: linee per um progetto di certificazione. Bollettino AIB, Roma, v. 39, n. 3, p. 245-71, 1999.

PERSPECTIVAS: o futuro da gestão da qualidade. Falando de qualidade, São Paulo, v. 14, n. 151, dez. 2004. p. 6-7.

PORTUONDO SÁNCHEZ, María Elena. ISO 9000: ¿Realidad o utopía en organizaciones de información? Ciencias de la Información, Habana, v. 29, n. 3, p. 53-56, sept. 1998.

RAMESH, L.S.R.C.V. Role of ISO 9000 standards in obtaining TQM in libraries: some problems and prospects. Herald of Library Science, Varanasi, v. 39, n. 1/2, p. 19-28, jan./apr. 2000.

SARDELLI, A. Il caso BNCF: la prima biblioteca italiana a impostare un sistema qualità conforme alle ISO 9000. Bibliotime Rivista Elettronica per le Biblioteche, Bologna, v. 4, n. 1, mar. 2001. Disponível em: <http://www.spbo.unibo.it/bibliotime/num-iv-1/sardelli.htm>. Acesso em: 21 dez. 2004.

. ISO 9000: tecnica della qualità e biblioteche: il percorso per ottenere la certificazione

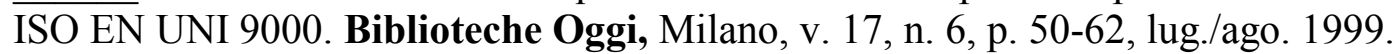

SELVI, G. T. Total Quality Management (TQM) and ISO 9000 in the context of Academic Library Services. SRELS Journal of Information Management, Bangalore, v. 39, n. 1, p. 105-115, mar. 2002.

TÉRMENS GRAELLS, M. Intranets para la gestión de una biblioteca: los flujos de información en un sistema de calidad ISO 9002. In: La gestión del conocimiento: retos y soluciones de los profesionales de la información. Bilbao: Servicio editorial de la Universidad del País Vasco, 2000. p. 607-612. Disponível em: <http://bd.ub.es/pub/termens/docs/intranetsiso.pdf>. Acesso em: 30 dez. 2004. 
VALLS, V. M. A aplicação da série ISO 9000 nos Serviços de Informação. In: CONGRESSO BRASILEIRO DE BIBLIOTECONOMIA, DOCUMENTAÇÃO E CIÊNCIA DA INFORMAÇÃO, 21., 2005, Curitiba. Anais... Curitiba: FEBAB, 2005.

VASSEUR, M. C. Au coeur de la démarche du certification ISO 9000: Le Centre de Documentation de la Société Grenobloise d'Études et d'Applications Hydrauliques. Bulletin des Bibliothèques de France, Paris, v. 43, n. 1, 1997. p. 44-46. Disponível em: $<$ http://www.enssib.fr/bbf/bbf-98-1/08-vasseur.pdf $>$. Acesso em: 30 dez. 2004.

VERGUEIRO, W. Qualidade em serviços de informação. São Paulo: Arte \& Ciência, 2002. $124 \mathrm{p}$.

WEDLAKE, L. J. An introduction to Quality Assurance and a guide to the implementation of BS 5750. Aslib Proceedings, London, v. 45, n. 1, p. 23-30, 1993.

\section{Valéria Martin Valls}

Doutora e Mestre em Ciências da Comunicação pela Escola de Comunicações e Artes da Universidade de São Paulo - ECA/USP; Docente do curso de Pós-Graduação da FESPSP (Fundação Escola de Sociologia e Política de São Paulo); Consultora associada da Terraforum Consultores.

e-mail: valls@uol.com.br

Agradecimentos:

Ao meu orientador Prof. Dr. Waldomiro Vergueiro.

Artigo aceito para publicação em janeiro de 2006. 